EGU21-13801, updated on 03 May 2021

https://doi.org/10.5194/egusphere-egu21-13801

EGU General Assembly 2021

(c) Author(s) 2021. This work is distributed under

the Creative Commons Attribution 4.0 License.

\title{
Impact induced electric field signals observed by the Solar Orbiter/RPW
}

Michiko Morooka ${ }^{1}$, Yuri Khotyaintsev ${ }^{1}$, Anders Eriksson ${ }^{1}$, Niklas Edberg ${ }^{1}$, Erik Johansson ${ }^{1}$, Milan Maksimovic ${ }^{2}$, Stuart Bale ${ }^{3,4}$, Thomas Chust ${ }^{5}$, Volodya Krasnoselskikh ${ }^{6}$, Matthieu Kretzschmar ${ }^{6,7}$, Eric Lorfèvre $^{8}$, Dirk Plettemeier ${ }^{9}$, Jan Souček ${ }^{10}$, Manfred Steller ${ }^{11}$, Štěpán Štverák ${ }^{12}$, Pavel Trávníček ${ }^{2,12}$, Andris Vaivads ${ }^{13}$, and Antonio Vecchio ${ }^{2,14}$

${ }^{1}$ Swedish Institute of Space Physics (IRFU), Uppsala, Sweden (morooka@irfu.se)

${ }^{2}$ LESIA, Observatoire de Paris, Université PSL, CNRS, Sorbonne Université, Univ. Paris Diderot, Sorbonne Paris Cité, 5 place Jules Janssen, 92195 Meudon, France

${ }^{3}$ Space Sciences Laboratory, University of California, Berkeley, CA, USA

${ }^{4}$ Physics Department, University of California, Berkeley, CA, USA

${ }^{5}$ LPP, CNRS, Ecole Polytechnique, Sorbonne Université, Observatoire de Paris, Université

${ }^{6} \mathrm{LPC2E}, \mathrm{CNRS}, 3 \mathrm{~A}$ avenue de la Recherche Scientifique, Orléans, France

${ }^{7}$ Université d'Orléans, Orléans, France

${ }^{8}$ CNES, 18 Avenue Edouard Belin, 31400 Toulouse, France

${ }^{9}$ Technische Universität Dresden, Würzburger Str. 35, D-01187 Dresden, Germany

${ }^{10}$ Institute of Atmospheric Physics of the Czech Academy of Sciences, Prague, Czechia

${ }^{11}$ Space Research Institute, Austrian Academy of Sciences, Graz, Austria

${ }^{12}$ Astronomical Institute of the Czech Academy of Sciences, Prague, Czechia

${ }^{13}$ Department of Space and Plasma Physics, School of Electrical Engineering and Computer Science, Royal Institute of Technology, Stockholm, Sweden

${ }^{14}$ Research Institute for Mathematics, Astrophysics and Particle Physics Radboud University, Nijmegen, The Netherlands

A large-amplitude impact-induced like electric field signal is often observed by the Radio and Plasma Wave (RPW) Instrument onboard Solar Orbiter. The signal has a sharp increase followed by an exponential decay, typically observed when spacecraft experiences a dust impact. The amplitude can reach several $\mathrm{V} / \mathrm{m}$. The impact dust size can be estimated from the electric field amplitude and is similar to the characteristic dust size near the sun expected from the zodiacallight observations. On the other hand, the signal's decay time is the order of second, unusually long compared to the dust impact signals previously reported by the other spacecraft. We will show the characteristics of these signals and discuss the origin. 